\title{
Kämpfen wie Che Guevara und Konkurrenz von Dr. Google
}

\section{Felicitas Witte}

Dr. med., Ärztin und Journalistin

Muss man als junger Hausarzt kämpfen wie Che Guevara? Oder sich Sorgen machen, von einem Roboter verdrängt zu werden? Berichtet wird von zwei Vorträgen: zum einen über die Diskussion zwischen einem erfahrenen Hausarzt und einem Berufseinsteiger. Nah am Patienten bleiben, darin waren sich die beiden einig. Zum anderen referierte eine junge Assistenzärztin und Forscherin über den aktuellen Stand in der «digitalen Gesundheitswelt».

Wie ein «alter Hase» und ein «frisch geschlüpfter» Hausarzt die Zukunft der Hausarztmedizin sehen, diskutierten Edy Riesen und Marius Bachofner. Riesen arbeitet seit mehr als 25 Jahren als Hausarzt in Ziefen BL, Bachofner arbeitet seit Anfang des Jahres in einer Praxis in Nottwil. Der Hausarzt, sagte Riesen, sei der Guerillero der Medizin - wie Che Guevara. "Als Hausarzt muss man fantasievoll sein und schlau», erklärte Riesen. «Wir sind ganz in der Nähe des Volkes, ganz nah bei den Patienten. Wir haben wie Guerilleros Utopien, für die wir kämpfen. Wir müssen wendig sein und uns in allen Fächern auskennen, vom Säugling bis zu Oma und Opa.»
Viele Kollegen würden klagen, weil Allgemeinärzte so wenig verdienen würden. «Ein Kardiologe verdient im zweiten Jahr 400000 Franken - das finde ich ungerecht», sagte Riesen. "Aber es ist immer die Frage, mit wem man sich vergleicht. Wir verdienen viel und können gut davon leben.» Man dürfe sich vielleicht auch nicht mit Kardiologen vergleichen, sagte Marius Bachofner. «Wir haben einen spannenden Job, und ich kann nach den ersten Monaten nur sagen: Es macht viel Spass.»

Edy Riesen liebt es, Fabeln zu erzählen, um das Leben als Arzt zu beschreiben. Zum Beispiel diese: Ein Hecht fragt zwei junge Fische: «Wie ist das Wasser?» Die

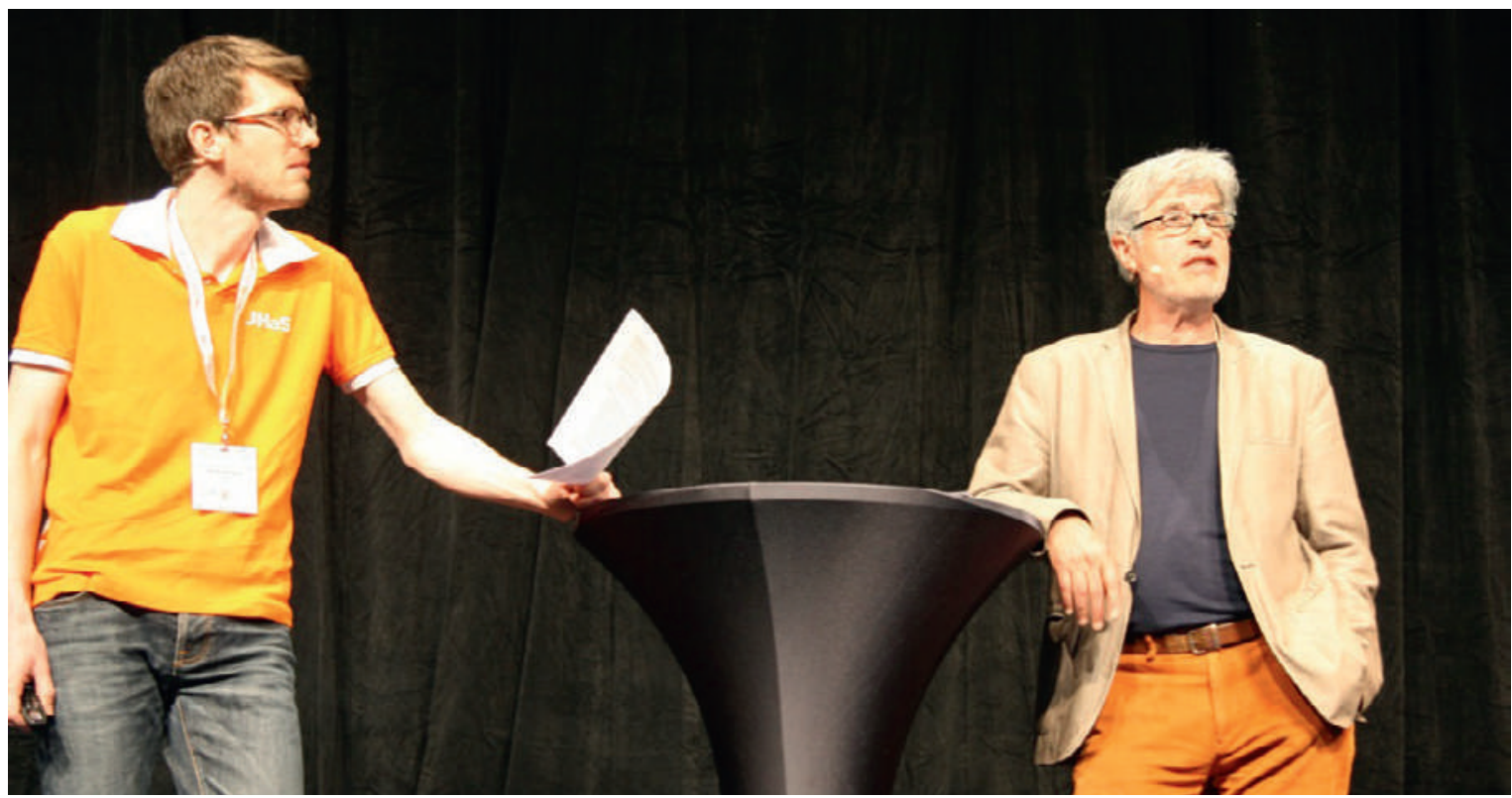

Der erfahrene Hausarzt Edy Riesen (rechts) und Marius Bachofner, der seit Januar 2016 als Hausarzt in Nottwil arbeitet, beleuchten das Metier des Hausarztes aus ganz unterschiedlichen Perspektiven. 
Fische schwimmen ohne Antwort weiter, und nach einer Weile fragt der eine den anderen: «Was meinte er eigentlich mit Wasser?» "Genauso wie die Fische das Wasser nicht mehr wahrnehmen, weil es sie ständig umgibt, nimmt man als Hausarzt das Verhältnis zum Patienten nicht wahr, weil es selbstverständlich ist und zum Leben dazu gehört», erklärte Riesen. "Es ist wie das Wasser für die Fische, und aus dem Kontext können wir uns nicht lösen.» Er hat viele Patienten schon als Kind gekannt und behandelt, kennt ihre Geschichten und die ihrer Familien. In seiner Praxis in Ziefen sieht er Patienten auch ausserhalb seiner Praxis fast täglich. Stirbt jemand im Dorf, begleitet er die Angehörigen. «Das Arbeiten wird dann zu einem kleinen Kosmos, wo alles andere draussen bleibt», erzählt er. Der Tod gehöre mit zu seinen schönsten Momenten als Arzt. «Es entsteht eine Intimität und eine Dankbarkeit, die man sonst nicht so erfährt.» Marius Bachofner erlebt den Kontakt mit seinen Patienten nicht mehr derart eng. «Wir jungen Ärzte wohnen nicht mehr so in der Nähe unserer Patienten - die Zeiten haben sich geändert.» Das wirke sich aber nicht unbedingt negativ auf die Beziehung zum Patienten aus. "Wir haben eine neue Kultur der Begegnung mit dem Patienten. Die ist vielleicht technischer und schneller, aber das muss nicht mit weniger Arzt-Patienten-Beziehung einhergehen.»

\section{Die grosse Stärke: Nähe zum Patienten}

Edy Riesen fällt immer wieder auf, wie sehr sich heutzutage die Patienten an Röntgen-, MRI-, CT- und andere Aufnahmen klammern. «Bilder haben eine ungeheure Verführungskraft», sagte er. «Die bildgebende Diagnostik wird heute aber total überbewertet, ebenso Laborwerte. Wenn der Patient vor einem sitzt und klagt, er habe Schmerzen, oder wenn er lacht und sagt, es ginge ihm gut - darauf achten leider einige Kollegen nicht.» Man müsse sich immer fragen, ob die Aufnahmen oder Tests wirklich notwendig seien. «Bilder führen zu einer gewissen Distanz zwischen Arzt und Patient», sagte Bachofner. «Bei der körperlichen Untersuchung sind wir dem Patienten dagegen viel näher.» Das Stethoskop sei die Legitimation, sich dem Patienten zu nähern. «Viele Kollegen lassen die Patienten sich gar nicht mehr entkleiden, sondern machen nur oberflächlich eine körperliche Untersuchung», erzählt er. "Aber wenn ich den Patienten richtig untersuche, bekomme ich viel mehr Informationen.» Auch Riesen muss sich eingestehen, dass er manchmal zuerst auf die Untersuchungsergebnisse schaut, so wie neulich bei dem Kleinkind mit Asthma, das zu einer Routinekontrolle kam. Er sah in der Lungenfunktion keine Än-

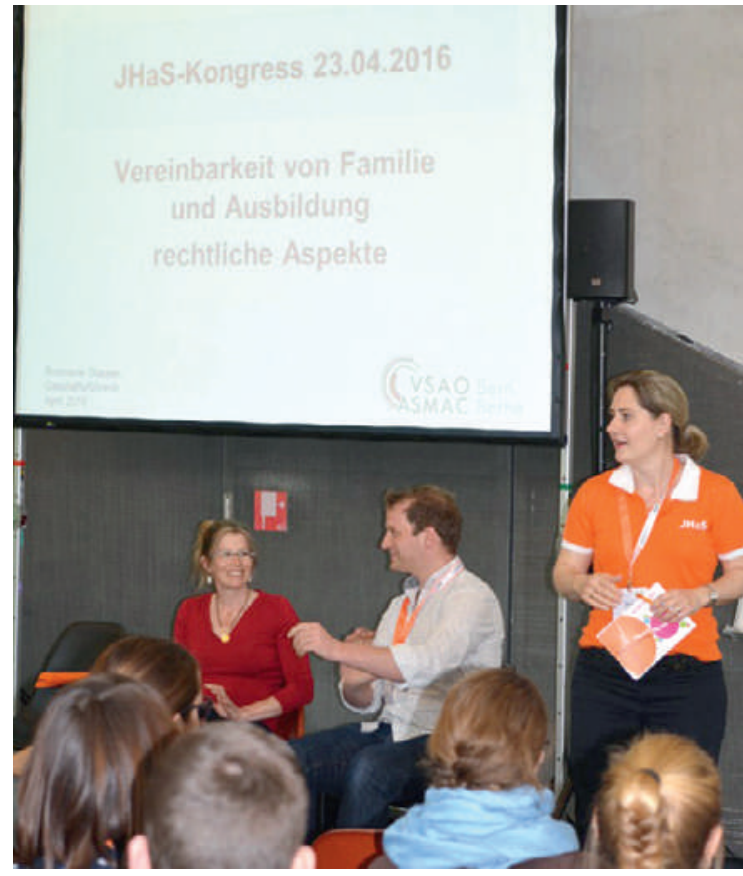

"Rechtliche Aspekte zur Vereinbarkeit von Beruf und Familie» war ein Thema von vielen, denen die Referenten nachgingen.

(Foto: JHaS)

derung und wollte Mutter und Kind gerade verabschieden - die Medikation müsse nicht geändert werden. «Der Kleine deutete dann aber auf mein Stethoskop und wollte, dass ich ihn abhöre», erzählt Riesen. «Er war unzufrieden, weil ich das vergessen hatte - und er hatte recht! Wir müssen uns täglich hinterfragen, ob das gut ist, was wir machen.»

Er habe viel durch Fehler und Niederlagen gelernt. «Wenn man im Notfall- oder Nachtdienst auf sich alleine gestellt ist, macht man manchmal Fehler, das ist ganz normal. Aber aus Fehlern kann man lernen und daran reifen.» Deshalb sehe er es auch kritisch, wenn manche Kollegen Nacht- und Notdienste für Hausärzte abschaffen wollten. Marius Bachofner hat den Eindruck, seine Generation würde sich manchmal zu sehr an starre Leitlinien klammern. «Die jüngeren Hausärzte haben oft den Wunsch nach Kochbuchmedizin. Wir haben aber die Kompetenzen und als Hausärzte die wunderbare Möglichkeit, eng am Patienten individuell auf ihn einzugehen. Das ist unsere grosse Stärke.»

\section{Konkurrenz von Dr. Google}

Das Motto des Kongresses lautete «get connected» - da liegt es nahe, auch über eHealth zu sprechen. Sima Djalali, Assistenzärztin und Forscherin am Institut für Hausarztmedizin in Zürich, gab einen prägnanten Überblick über den aktuellen Stand der «digitalen 


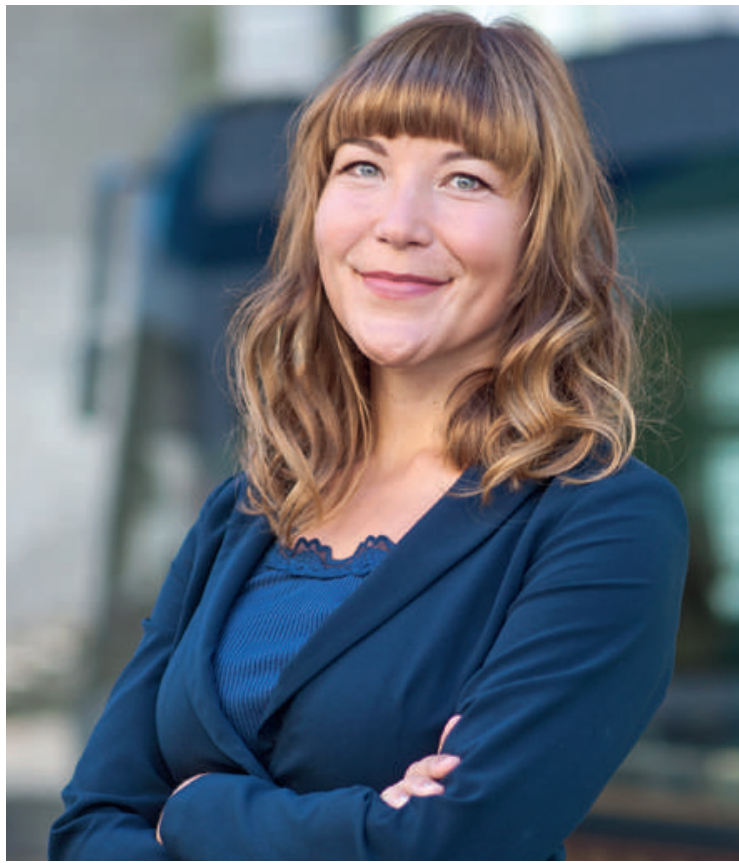

Was müssen Ärzte über die "digitale Gesundheitswelt» wissen? Sima Djalali, Assistenzärztin und Forscherin am Institut für Hausarztmedizin in Zürich, gab einen Einblick in das Thema "eHealth». (Foto: $z V g$ von Sima Djalali)

Gesundheitswelt» und was Ärzte dazu wissen müssen. Viele Patienten googeln inzwischen ihre Diagnose im Internet. «Frau Doktor, ich habe Diabetes», so komme der Patient dann in die Praxis, erzählte Djalali. Der Patient, ein 34-jähriger Mann mit einem BMI von 24 ohne Vorerkrankungen fühlte sich müde und abgeschlagen, klagte über trockene Haut im Gesicht, und sein hartnäckiger Fusspilz besserte sich trotz einmonatiger Therapie nicht. Der Internetdoktor hatte die Diagnosen «Diabetes» oder «Woodhouse Sakati-Syndrom» gestellt. «Weder das eine noch das andere trifft natürlich zu», sagte Djalali. «Dr. Google ist nicht so gut wie viele meinen.» Nur in 34 Prozent der Fälle stellt der Internetdoktor die korrekte Diagnose [1]. In 58 Prozent der Fälle listet er diese unter den ersten 20 möglichen Diagnosen auf. Immerhin erkannte der digitale Kollege 80 Prozent der Notfälle richtig. «Man darf daraus aber nicht schliessen, dass eHealth nichts bringt», sagte Djalali. «Richtig eingesetzt kann eHealth bei Vorbeugung, Diagnose, Behandlung und Überwachung im Gesundheitswesen viel helfen.» Djalali unterschied verschiedene «Reifestufen» von eHealth. eHealth-Anwendungen der tiefsten Reifestufe dienen dazu, Abläufe im Gesundheitswesen zu unterstützen, etwa indem Informationen elektronisch bereitgestellt werden. Wer im Internet nach Symptomen googelt, nutzt diese einfachste Form von eHealth. «Wenn man nach Informationen sucht, muss man genau darauf achten, wer hin- ter den Seiten steckt», sagte Djalali. Seiten mit dem HONcode seien in der Regel seriös [2]. Man kann sich die HONcode-Symbolleiste mit ein paar Klicks einfach installieren [3]. Ist eine Seite HON-zertifiziert, erkennt man das am kleinen blau-roten Symbol am oberen Bildschirmrand. «Wie man seriöse Gesundheitsseiten findet, sollten wir auch den Patienten erklären», riet Djalali. «Bei dem jungen Mann, der meinte, Diabetes zu haben, würde ich mich erst einmal erkundigen, welchen Internetdoktor er gefragt hat.»

Ein Beispiel für eHealth-gestützte Kommunikation im Gesundheitswesen ist die «Online-Praxis», ein Service, der von einem Schweizer Unternehmen zusätzlich zur elektronischen Krankengeschichte angeboten wird. Patienten bekommen dabei Zugang auf eine geschützte Onlineplattform, über die sie ihrem Arzt eine Nachricht schicken und Fragen stellen, Symptome schildern oder Fotos senden können. Der Arzt wird darüber per SMS informiert und kann via Onlineplattform antworten. Eine Kopie der Kommunikation landet automatisch in der elektronischen Krankengeschichte. Ein anderes Beispiel sind digitale Patiententagebücher, etwa bei Diabetes oder Bluthochdruck. Per Mobiltelefon oder am Computer trägt der Patient regelmässig seine Werte ein, die via Internet in die digitale Krankenakte des Arztes gelangen. «Das ist sehr praktisch, das kann man gemeinsam mit dem Patienten anschauen und die Therapiestrategie anpassen", sagte Djalali.

\section{Von eHealth-Interaktion und dem Potential junger Hausärzte}

Findet die elektronisch unterstützte Kommunikation zwischen Arzt und Patient in Echtzeit statt, ist von eHealth-Interaktion die Rede - etwa per Videokonsultation. In der Schweiz bieten Apotheken das zum Beispiel unter dem Begriff «netCare» in Zusammenarbeit mit dem Telemedizinanbieter Medgate an. «Man muss aber nicht unbedingt mit einem Arzt interagieren", sagte Djalali. Bei der Raucher-Hilf-App «Smoke Free Buddy" ist man zum Beispiel in engem Kontakt mit einem «Buddy», einem Ex-Raucher [4]. Der unterstützt einen mit aufmunternden Worten und Tipps gegen das akute Verlangen nach einer Zigarette und steht für Chats zur Verfügung.

Höher entwickelte eHealth-Anwendungen dienen der Dokumentation und dem gezielten Datenaustausch, etwa elektronische Krankengeschichten und OnlinePatientendossiers. Der Bundesrat hatte im Mai 2013 den Gesetzesentwurf über das elektronische Patientendossier (EPDG) verabschiedet und an das Parlament überwiesen. Im Juni 2015 stimmten beide Räte der Vor- 


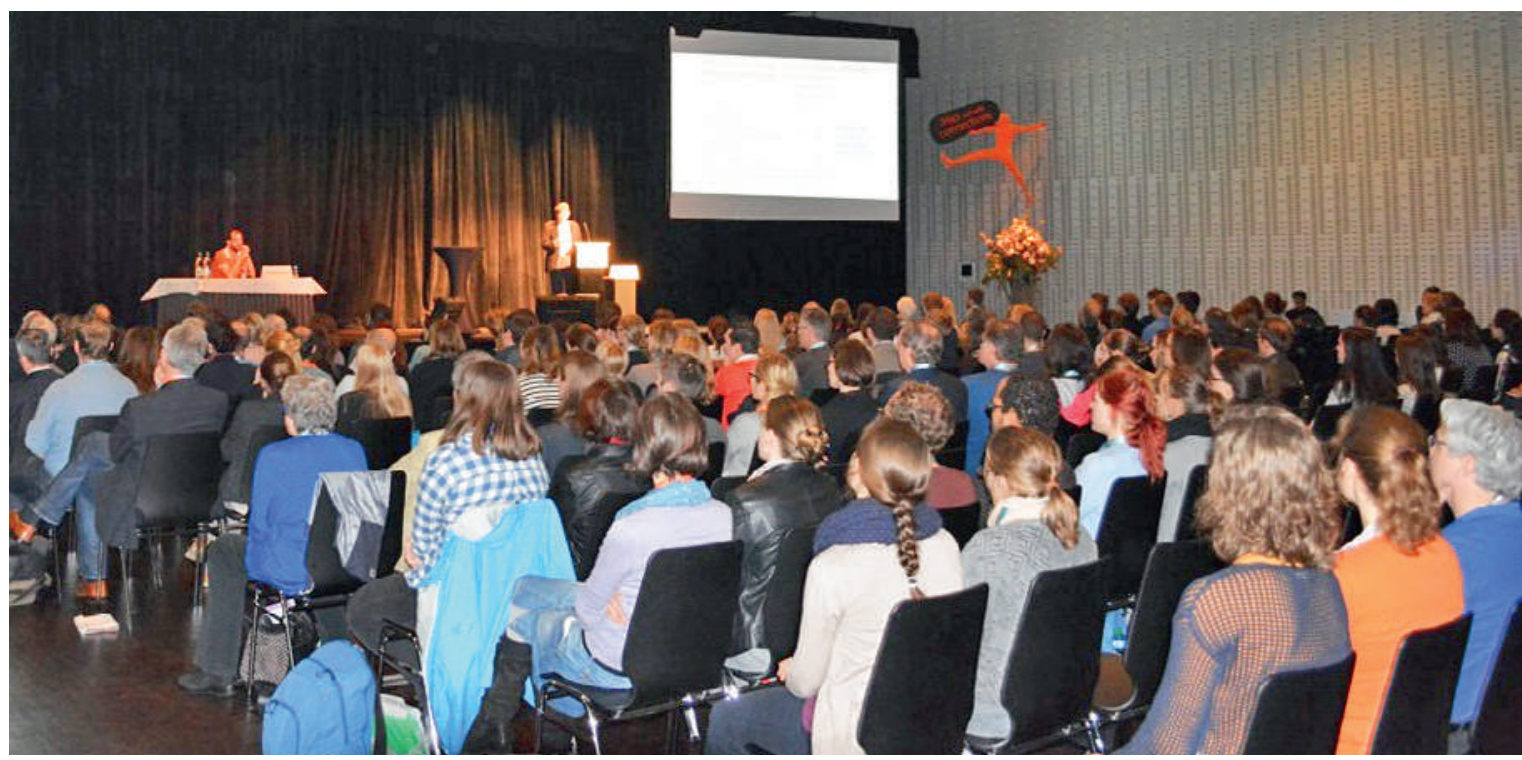

Gespannt verfolgten die Kongressteilnehmer die Vorträge rund um verschiedene Themen ihres beruflichen Alltags. (Foto: JHaS)

lage des Entwurfs zu. Gemäss dem Beschluss sind die Spitäler verpflichtet, nach einer Übergangsfrist von drei Jahren am System teilzunehmen, die Pflegeheime nach fünf Jahren. Für alle Behandelnden im ambulanten Bereich bleibt die Teilnahme freiwillig. Auch die Patienten entscheiden, ob sie mitmachen wollen oder nicht. Kommerzielle Angebote gibt es bereits heute, beispielsweise von der Swisscom [5]. «Elektronische Dossiers funktionieren, aber nur, wenn alle beim selben Dossier mitmachen und keine Insellösungen betrieben werden", sagte Djalali. Bei den kommerziellen Dossiers wird auch der Lebensstil erfasst, etwa wie viel man sich bewegt, was man isst und trinkt. «Das ist der nächste Schritt: nicht Kranken-, sondern GesundheitsAkte», sagte Djalali. "Der Kühlschrank misst, was wir essen, die Waage registriert das Gewicht, das Fitnessarmband die Bewegung, und alle sagen gemeinsam: Hey, du solltest dich mal wieder bewegen und gesünder essen", skizzierte Djalali ein Zukunftsszenario.

Die reifste Stufe von eHealth zum gegenwärtigen Zeitpunkt ist Big Data. Das sind Massendaten, die viel zu gross und zu inhomogen sind, um sie mit den derzeit zur Verfügung stehenden technischen Mitteln zu bündeln. Riesencomputer suchen nach Korrelationen, was bei der Hypothesengenerierung helfen könnte und konsequenterweise bei der Entwicklung neuer Therapien. «Das könnte die Medizin revolutionieren - aber bislang weiss niemand wirklich damit umzugehen.» Bis jetzt hat die Ärztin keine Angst, durch «Dr. Google» ersetzt zu werden. «Gerade in den USA, wo man sehr leicht verklagt werden kann, möchte niemand wegen seines Symptomcheckers in die Schlagzeilen geraten, weil Dr. Google sagte: Dein Problem ist nicht schlimm, bleib zu Hause.» Die Organisatoren solcher Seiten seien vorsichtig und würden im Zweifel immer raten, einen «echten Arzt» aufzusuchen. «Solange unsere Internetkollegen so vorsichtig sind, brauchen wir keine Sorge haben, durch Roboter ersetzt zu werden.»

Einen eigenen Kongress für junge Hausärzte bräuchte es rein fachlich eigentlich nicht, findet Manuel Schaub, angehender Hausarzt in Lenzburg AG. "Aber so eine Tagung ist wichtig, damit angehende Hausärzte sehen, dass sie mit ihrem Berufswunsch nicht alleine sind.» Ihm habe die Tagung viel Spass gemacht, und sie habe ihm fachlich etwas gebracht. «Die Organisatoren haben nicht auf «krampfhaft jugendlich` gemacht, sondern waren einfach so, wie wir jungen Ärzte sind», erzählt er. «Ich fand super, selbstbewusste Hausärzte und Hausärztinnen mit Ausstrahlung zu sehen.» Oft meine man als junger Hausarzt, man tauche ein in eine Welt von nur älteren Kollegen. «Dem ist aber nicht so. Wir sind viele, wir sind jung, und wir haben ein grosses Potential!»

\section{Literatur}

1 Semigran HL, et al. BMJ. 2015;351:h3480.

2 http://www.hon.ch/HONcode/Patients/Visitor/visitor_de.html

3 https://addons.mozilla.org/de/firefox/addon/hon-toolbar/

4 http://www.smokefree.ch/en/buddy-app/1/

5 https://www.evita.ch/de/main/home 